\title{
Geometric quotients of unipotent group actions II
}

\author{
Gert-Martin Greuel \\ Universität Kaiserslautern \\ Fachbereich Mathematik \\ Erwin-Schrödinger-Strasse \\ 67663 Kaiserslautern \\ GERMANY
}

\author{
Gerhard Pfister \\ Universität Kaiserslautern \\ Fachbereich Mathematik \\ Erwin-Schrödinger-Strasse \\ 67663 Kaiserslautern \\ GERMANY
}

\section{Introduction}

Let $G$ be a unipotent algebraic group over $K$ (a field of characteristic 0 ) which acts rationally on an affine scheme $X=\operatorname{Spec} A$ over $K$, where $A$ is a commutative $K$-algebra. The problem of finding sufficient and manageable conditions to guarantee that the geometric quotient $X / G$ exists is of fundamental interest in the theory of moduli spaces for local objects such as isolated singularities or (Cohen-Macaulay) modules over the local ring of a singularity (cf. [L-P], [G-P2], [G-H-P], [H]).

In [G-P1] we derived such conditions which are complemented in this paper. These conditions are even useful when the geometric quotient does not exist globally. Namely, they allow the construction of a stratification of $X$ into locally closed $G$-stable subschemes on which the geometric quotient exists. If the action of $G$ is sufficiently explicitly given, say in terms of coordinates of $X$ and generators of $G$, then the stratification can be described explicitly in terms of these data. Note that for unipotent groups, in contrast to reductive groups, the existence of a geometric quotient depends in general not only on $X$ and $G$ but also on the action, that is, knowledge about the action is necessary. The purpose of [G-P1] was to prove existence criteria which were as general and as explicit as possible. In all applications so far, the explicit description of the strata was the key point to being able to describe the strata in terms of invariants of the singularities or modules.

On the other hand, the explicit formulation in terms of coordinates and generators made the statements of the theorems in [G-P1] somewhat technical, even in the case of a free action, which is an important cornerstorne for the general theory (cf. Theorem 3.10 in [G-P1]). One of the equivalent conditions of that theorem (loc. cit.) was the vanishing of $H^{1}(G, A)$ (the usual algebraic 
group cohomology), in particular we showed that $H^{1}(G, A)=0$ is equivalent to Spec $A \longrightarrow \operatorname{Spec} A^{G}$ being a trivial geometric quotient (which implies in particular that $A^{G}$ is of finite type over $K$ if $A$ is of finite type over $K$ ). Moreover, we proved that $H^{1}(G, A)=0$ implies that $\operatorname{Spec} A \longrightarrow \operatorname{Spec} A^{G}$ is a principal fibre bundle with group $G$, that is, $\operatorname{Spec} A \rightarrow \operatorname{Spec} A^{G}$ is faithfully flat and the canonical map $A \otimes_{A^{G}} A \longrightarrow A \otimes_{K} K[G]$ is an isomorphism (cf. [M-F], Def. 0.10).

In this note, which is intended to be a supplement of [G-P1], we prove the converse of the last statement, providing the following conceptual, necessary and sufficient condition for $H^{1}(G, A)=0$.

Theorem: Let $A$ be a commutative $K$-algebra and $G$ a unipotent algebraic group over $K$ acting rationally on Spec $A$. Then the following are equivalent:

(i) $H^{1}(G, A)=0$;

(ii) Spec $A \longrightarrow \operatorname{Spec} A^{G}$ is faithfully flat and the canonical map

(*) $\quad A \otimes_{A^{G}} A \longrightarrow A \otimes_{K} K[G]$ is an isomorphism.

Moreover, if $A$ is reduced, then (i) and (ii) are equivalent to

(ii') Spec $A \longrightarrow \operatorname{Spec} A^{G}$ is faithfully flat and the canonical map

(**) $\quad A \otimes_{K} A \longrightarrow A \otimes_{K} K[G]$ is surjective.

Condition (**) means that $X \times_{K} G \longrightarrow X \times_{K} X$ is a closed immersion, that is, the action is free in the sense of Mumford (cf.[M-F], Def. 0.8). We ignore whether we can drop the assumption of $A$ being reduced in (ii'). Note that $(*)$ implies $(* *)$ but that $(*)$ does not imply the flatness of $A$ over $A^{G}$, cf. the example in [D-F], examined at the end of this paper.

That (i) implies (ii) follows from [G-P1], Theorem 3.10 and Remark 3.11; (ii') is a trivial consequence of (ii). The remaining implications are proved in this paper.

The equivalence of (i) and (ii) was already mentioned in [K-M-T], but some arguments in the proof seemed to be insufficient. More recently, in [D-F-G], a result was proved which states (in our terms) the implication (ii) $\Rightarrow$ (i) for $G$ the additive group of $K=\mathbb{C}$ and $A$ the polynomial ring over $\mathbb{C}$. In any case, here we give an elementary proof of the following slightly more general fact.

If $\left(^{*}\right)$ holds, and if the canonical map $\operatorname{Spec} A \longrightarrow \operatorname{Spec} A^{G}$ is flat, then $\operatorname{Spec} A$ is mapped onto an open set $U \subset \operatorname{Spec} A^{G}$ such that Spec $A \rightarrow U$ is a geometric quotient and a principal fibre bundle with group $G$. 
This article was inspired by discussions with C. Hertling, when we tried to extend the results of [G-H-P], in order to construct moduli spaces for semiquasihomogeneous hypersurface singularities without fixing the principal part. We could not prove the existence of a geometric quotient as an algebraic $\mathbb{C}$-scheme. From the examples of Deveney and Finston we learned that, additionally, at least the flatness of $A$ as an $A^{G}$-module is necessary. Condition (ii') shows that it is also sufficient if $A$ is reduced. Although we could not prove the existence of a geometric quotient as an algebraic $\mathbb{C}$-variety under the assumption "Spec $A \longrightarrow \operatorname{Spec} A^{G}$ surjective and (**) holds", in our application Hertling was able to prove the existence of a geometric quotient as a complex space.

The following conjecture points in the same direction ( $G$ and $A$ as above):

Conjecture. Assume that $G$ acts freely on $\operatorname{Spec} A$ (in the sense of Mumford). Then there exists an étale covering $\left\{\operatorname{Spec} B_{i}\right\}$ of $\operatorname{Spec} A$ and a lifting of the action of $G$ to $B_{i}$ such that $H^{1}\left(G, B_{i}\right)=0$.

Notice that under our assumption the quotient exists in the category of algebraic spaces (cf. [P, Theorem 3.7]). Our conjecture says that this quotient is locally trivial. We prove this under a slightly different assumption. We should like to emphasize that passing to an étale covering is necessary, as we show at the end of this paper.

As in [G-P1] we prefer to work with the Lie algebra $L$ of $G$. Since $G$ is unipotent and char $K=0$ this is equivalent. Also the Lie algebra cohomology ([C-E]) coincides in this case with the group cohomology.

\section{Special representations}

Let $L$ be an $n$-dimensional nilpotent $K$-Lie algebra. We deduce the vanishing of $H^{1}\left(L, K\left[X_{1}, \ldots, X_{n}\right]\right)$ for certain special representations of $L$ in $\operatorname{der}_{K} A\left[X_{1}, \ldots, X_{n}\right]$, in particular for the representation of $L$ on the coordinate algebra $K[G(L)] \cong K[L]=K\left[X_{1}, \ldots, X_{n}\right]$ of its associated unipotent group $G(L)$ derived from the left regular representation of $G(L)$ on $K[G(L)]$. This result is perhaps known to the specialists but we could not find a reference. In any case, it is an immediate consequence of Theorem 3.10 in [G-P1]. In order to apply that theorem we need a description of the left regular action in terms of coordinates.

Let $X, Y \in L$ be two elements and $H(X, Y)=\sum_{i>0} H^{i}(X, Y)$ the series of Campbell-Hausdorff (cf. [G]), where

$$
\begin{aligned}
H^{1}(X, Y) & =X+Y \\
H^{2}(X, Y) & =\frac{1}{2}[X, Y] \\
H^{3}(X, Y) & =\frac{1}{12}([X,[X, Y]]+[Y,[Y, X]]), \ldots
\end{aligned}
$$

and $H^{i}(X, Y)=0$ for large $i$ since $L$ is nilpotent. 
Consider $L$ as an affine $K$-variety. Then the multiplication $H: L \times L \longrightarrow L$ gives $L$ the structure of a unipotent algebraic group which we call $G(L)$, with Lie-algebra isomorphic to $L$ (cf. [D-G]).

Let $\left\{\delta_{1}, \ldots, \delta_{n}\right\}$ be a basis of $L$ and $\left[\delta_{i}, \delta_{j}\right]=\sum_{k} C_{k}^{i j} \delta_{k}$. The choice of a basis defines an isomorphism $G(L) \cong \operatorname{Spec} K\left[X_{1}, \ldots, X_{n}\right]$ and then the comultiplication

$$
m: K\left[X_{1}, \ldots, X_{n}\right] \longrightarrow K\left[X_{1}, \ldots, X_{n}\right] \otimes_{K} K\left[X_{1}, \ldots, X_{n}\right],
$$

is given in terms of the chosen coordinates by

$$
m\left(X_{k}\right)=X_{k} \otimes 1+1 \otimes X_{k}+\frac{1}{2} \sum_{i j} C_{k}^{i j} X_{i} \otimes X_{j}+\ldots
$$

Via $G(L) \cong$ Spec $K\left[X_{1}, \ldots, X_{n}\right]$ the Lie-algebra $L$ is represented as a subalgebra of $\operatorname{Der}_{K} K\left[X_{1}, \ldots, X_{n}\right]$ with basis $\left\{\delta_{j}\right\}$ and $\delta_{j}\left(X_{k}\right)=\delta_{j k}+\sum_{i} C_{k}^{i j} X_{i}+h_{j k}$ $\left(\delta_{j k}\right.$ the Kronecker symbol, $\left.h_{j k} \in\left(X_{1}, \ldots, X_{n}\right)^{2}\right)$, the derived left regular representation of $L$ on $K[G(L)] \cong K[L]=K\left[X_{1}, \ldots, X_{n}\right]$.

Since $L$ is nilpotent, we can choose the basis $\left\{\delta_{1}, \ldots, \delta_{n}\right\}$ such that $C_{k}^{i j}=0$ if $k \leq \max \{i, j\}$. This implies that $L$ acts on $K[L]$ via

1. $\delta_{j}\left(X_{j}\right)=1$,

2. $\delta_{j}\left(X_{k}\right)=0$ if $j>k$,

3. $\delta_{j}\left(X_{k}\right) \in K\left[X_{1}, \ldots, X_{k-1}\right]$ if $j<k$

and, in particular,

3.' $\delta_{i} \delta_{j}\left(X_{k}\right)=0$ if $i \geq k$.

Using Theorem 3.10 in [G-P1], we obtain the following

Proposition 1.1 The derived left regular representation of $L$ on $K[L]$ satisfies $H^{1}(L, K[L])=0$.

Corollary 1.2 Let $L$ be an $n$-dimensional nilpotent $K$-Lie-algebra. There exists a faithful representation $\rho: L \longrightarrow \operatorname{Der}_{K} K\left[X_{1}, \ldots, X_{n}\right]$ such that

$$
H^{1}\left(L, K\left[X_{1}, \ldots, X_{n}\right]\right)=0 .
$$

Corollary 1.3 Let $A$ be a commutative $K$-algebra (with unit 1), $L$ an $n$ dimensional nilpotent $K$-Lie-algebra and $\varphi: L \longrightarrow \operatorname{Der}_{K} A$ a representation such that the elements of $\varphi(L)$ are locally nilpotent. Let $\rho: L \longrightarrow$ $\operatorname{Der}_{K} K\left[X_{1}, \ldots, X_{n}\right]$ be any representation satisfying

$$
H^{1}\left(L, K\left[X_{1}, \ldots, X_{n}\right]\right)=0 .
$$

Then for the tensor-product representation $\varphi \otimes \rho: L \longrightarrow \operatorname{Der}_{K} A\left[X_{1}, \ldots, X_{n}\right]$ we have 
(i) $H^{1}\left(L, A\left[X_{1}, \ldots, X_{n}\right]\right)=0$.

(ii) Let $\left\{\delta_{1}, \ldots, \delta_{n}\right\}$ be a basis of $L$ such that $\left[\delta_{i}, \delta_{j}\right] \in \sum_{\ell>\max \{i, j\}} K \delta_{\ell}$ (such a basis does always exist) then $\left(\exp \left(-X_{1} \delta_{1}\right) \circ \cdots \circ \exp \left(-X_{n} \delta_{n}\right)\right)(A)=$ $A\left[X_{1}, \ldots, X_{n}\right]^{L}$.

(iii) Consider a basis $\left\{\delta_{1}, \ldots, \delta_{n}\right\}$ of $L$ as in (ii) and extend it trivially to $A\left[X_{1}, \ldots, X_{n}\right]$ (that is $\delta_{j}\left(X_{i}\right)=0$ ), then

$$
\begin{aligned}
& \delta_{i} \circ \exp \left(-X_{1} \delta_{1}\right) \circ \cdots \circ \exp \left(-X_{n} \delta_{n}\right) \\
& \quad=\exp \left(-X_{1} \delta_{1}\right) \circ \cdots \circ \exp \left(-X_{n} \delta_{n}\right) \circ\left(\delta_{i}+\sum_{k>i} \xi_{i_{k}} \delta_{k}\right)
\end{aligned}
$$

for suitable $\xi_{i_{k}} \in K\left[X_{1}, \ldots, X_{n}\right]$.

Proof. The first claim follows because $H^{1}\left(L, K\left[X_{1}, \ldots, X_{n}\right]\right)=0$.

To prove (ii) let $\hat{\delta}_{i}=\rho \otimes \varphi\left(\delta_{i}\right)$. Denote by

$$
s: A\left[X_{1}, \ldots, X_{n}\right] \longrightarrow A\left[X_{1}, \ldots, X_{n}\right]^{L}
$$

the section (cf. [G-P1]) defined by

$$
s(h)=\left(\exp T_{1} \hat{\delta}_{1} \circ \cdots \circ \exp T_{n} \hat{\delta}_{n}\right)(h)\left(T_{1}=-X_{1}, \ldots, T_{n}=-X_{n}\right),
$$

where $\left(T_{1}=-X_{1}, \ldots, T_{n}=-X_{n}\right)$ means evaluation at $T_{i}=-X_{i}$.

If $a \in A$ then $\hat{\delta}_{i}(a)=\delta_{i}(a)$. This implies $s(a)=\left(\exp \left(-X_{1} \delta_{1}\right) \circ \cdots \circ\right.$ $\left.\exp \left(-X_{n} \delta_{n}\right)\right)(a)$, that is

$$
\exp \left(-X_{1} \delta_{1}\right) \circ \cdots \circ \exp \left(-X_{n} \delta_{n}\right)(A) \subseteq A\left[X_{1}, \ldots, X_{n}\right]^{L} .
$$

If $h=a+\sum X_{i} h_{i}, a \in A$, then $s(h)=s(a)$ because $s\left(X_{i}\right)=0$. This proves (ii). Since $\left[\delta_{i}, \delta_{j}\right] \in \sum_{\ell>\max \{i, j\}} K \delta_{\ell}$ (iii) holds.

The following corollary points towards the conjecture in the introduction. It is an improvement of Remark 3.12 of [G-P1], where we assumed that $L$ is abelian. Note that $\operatorname{det}\left(\delta_{i}\left(a_{j}\right)\right) \in A^{*}$ implies that the action is set theoretically free.

Corollary 1.4 Let $A$ be a commutative $K$-algebra with 1 and $L \subset \operatorname{der}_{K}(A)$ an $n$-dimensional nilpotent $K$-Lie algebra. Assume that there exist $\delta_{1}, \ldots, \delta_{n} \in L$ and $a_{1}, \ldots, a_{n} \in A$ such that $\operatorname{det}\left(\delta_{i}\left(a_{j}\right)\right)$ is a unit in $A$. Let $X_{1}, \ldots, X_{n}$ be indeterminates and define $F_{i}:=\exp \left(-X_{1} \delta_{1}\right) \circ \cdots \circ \exp \left(-X_{n} \delta_{n}\right)\left(a_{i}\right)$. Then

$$
B:=A\left[X_{1}, \ldots, X_{n}\right] /\left(F_{1}, \ldots, F_{n}\right)
$$

is étale over $A$, the action of $L$ on $A$ lifts to $B$ and $H^{1}(L, B)=0$. 
Proof. By Corollary 1.3 there exists a faithful representation of $L$ such that $H^{1}\left(L, K\left[X_{1}, \ldots, X_{n}\right]\right)=0$. Using any such representation, we define the tensor product representation of $L$ on $A\left[X_{1}, \ldots, X_{n}\right]$ as in 1.3. Then the $F_{i}$ are invariant under $L$ by 1.3 (ii). The vanishing of $H^{1}(L, B)$ is now a consequence of 1.3(i) and Theorem 3.10 in [G-P1].

To prove that $A \rightarrow B$ is étale, we may assume that $\delta_{1}, \ldots, \delta_{n}$ are chosen as in (ii) of 1.3 and $\hat{\delta}_{1}, \ldots, \hat{\delta}_{n} \in \operatorname{Der}_{K} A\left[X_{1}, \ldots, X_{n}\right]$ are extensions of $\delta_{1}, \ldots, \delta_{n}$ such that $\hat{\delta}_{i}\left(F_{j}\right)=0$ for all $i, j$. Let $\bar{\delta}_{i}:=\delta_{i}-\hat{\delta}_{i}, i=1 \ldots n$ (the $\delta_{i}$ are trivially extended to $\left.A\left[X_{1}, \ldots, X_{n}\right]\right)$; then $\bar{\delta}_{i} \in \operatorname{Der}_{A} A\left[X_{1}, \ldots, X_{n}\right]$.

Furthermore, by 1.3 (iii),

$$
\begin{aligned}
\bar{\delta}_{i}\left(F_{j}\right) & =\delta_{i}\left(F_{j}\right) \\
& =\delta_{i} \circ \exp \left(-X_{1} \delta_{1}\right) \circ \cdots \circ \exp \left(-X_{n} \delta_{n}\right)\left(a_{j}\right) \\
& =\exp \left(-X_{1} \delta_{1}\right) \circ \cdots \circ \exp \left(-X_{n} \delta_{n}\right)\left(\delta_{i}\left(a_{j}\right)+\sum_{k>i} \xi_{i_{k}} \delta_{k}\left(a_{j}\right)\right) .
\end{aligned}
$$

Then

$$
\begin{aligned}
\operatorname{det}\left(\bar{\delta}_{i}\left(F_{j}\right)\right) & =\exp \left(-X_{1} \delta_{1}\right) \circ \cdots \circ \exp \left(-X_{n} \delta_{n}\right)\left(\operatorname{det}\left(\delta_{i}\left(a_{j}\right)+\sum_{k>i} \xi_{i_{k}} \delta_{k}\left(a_{j}\right)\right)\right) \\
& =\exp \left(-X_{1} \delta_{1}\right) \circ \cdots \circ \exp \left(-X_{n} \delta_{n}\right)\left(\operatorname{det}\left(\delta_{i}\left(a_{j}\right)\right)\right) \\
& =\operatorname{det}\left(\delta_{i}\left(a_{j}\right)\right) .
\end{aligned}
$$

This implies that $\operatorname{det}\left(\bar{\delta}_{i}\left(F_{j}\right)\right)$ is a unit and, therefore,

$$
A \rightarrow A\left[X_{1}, \ldots, X_{n}\right] /\left(F_{1}, \ldots, F_{n}\right)
$$

is étale.

\section{Free actions}

We are now going to prove the main theorem which was explained in the introduction.

Theorem 2.1 Let $A$ be a commutative $K$-algebra. Let $L=\sum_{i=1}^{n} K \delta_{i} \subseteq$ $\operatorname{Der}_{K}(A)$ be an $n$-dimensional nilpotent Lie-algebra and assume that the $\delta_{i}$ are locally nilpotent. Assume, moreover, that

1. Spec $A \longrightarrow$ Spec $A^{L}$ is faithfully flat,

2. the canonical map $A \otimes_{A^{L}} A \longrightarrow A\left[Z_{1}, \ldots, Z_{n}\right]$ defined by $L$,

$$
a \otimes b \rightsquigarrow a \cdot\left(\exp Z_{1} \delta_{1} \circ \dot{s} \circ \exp Z_{n} \delta_{n}\right)(b)
$$

is an isomorphism.

Then $H^{1}(L, A)=0$. 
Proof. We prove the theorem by induction on $n=\operatorname{dim} L$. In the case $n=1$ let $L=K \delta$ and $\int A^{L}=\left\{a \in A \mid \delta(a) \in A^{L}\right\}$.

The sequence

$$
0 \longrightarrow \int A^{L} \longrightarrow A \stackrel{\delta^{2}}{\longrightarrow} A
$$

is exact. Let $L$ act on $A[Z]$ by $\delta(Z):=-1$, then $B:=\exp (-Z \delta)(A) \subseteq A[Z]^{L}$, and $B$ is via $A^{L} \subseteq A \stackrel{\sim}{\longrightarrow} B$ an $A^{L}$-algebra. Since $B$ is flat over $A^{L}$,

$$
0 \longrightarrow \int A^{L} \otimes_{A^{L}} B \longrightarrow A \otimes_{A^{L}} B \stackrel{\delta^{2} \otimes 1_{B}}{\longrightarrow} A \otimes_{A^{L}} B
$$

is exact.

By assumption, $A \otimes_{A^{L}} A \longrightarrow A[Z]$ is an isomorphism, that is, we may identify $A \otimes_{A^{L}} B$ with $A[Z]$ and obtain the commutative diagram:

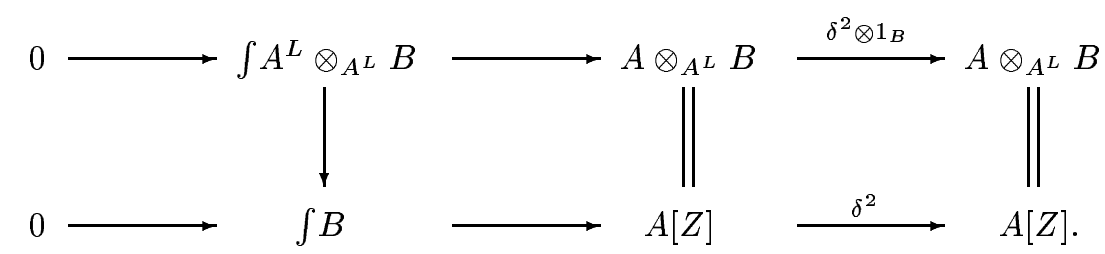

Also by assumption $Z=\sum \xi_{i} h_{i}, \xi_{i} \in A, h_{i} \in B$. On the other hand, $\delta(Z)=$ -1 whence $\delta^{2}(Z)=0$. This implies that we can choose the $\xi_{i}$ to be in $\int A^{L}$, by the above diagram.

Then $-1=\delta(Z)=\sum \delta\left(\xi_{i}\right) h_{i}$ and, in particular,

$$
-1=\sum \delta\left(\xi_{i}\right) h_{i}(Z=0) .
$$

Now $\delta\left(\xi_{i}\right) \in A^{L}$ and $h_{i}(Z=0) \in A$. If $\mathfrak{a}=\left\langle\left\{\delta\left(\xi_{i}\right)\right\}_{i}\right\rangle$ denotes the $A^{L}$-ideal generated by the $\delta\left(\xi_{i}\right)$ in $A^{L}$, then $\mathfrak{a} A=A$. By faithful flatness we have $\mathfrak{a}=A^{L}$, that is there are $\eta_{i} \in A^{L}$ such that $1=\sum \delta\left(\xi_{i}\right) \eta_{i}=\delta\left(\sum \xi_{i} \eta_{i}\right)$.

If we define $x:=\sum \xi_{i} \eta_{i} \in A$ then $\delta(x)=1$ and this implies $H^{1}(L, A)=0$ ([G-P1], 3.10).

Now assume the theorem for $(n-1)$-dimensional Lie-algebras. Let $L=$ $\sum_{i=1}^{n} K \delta_{i}$ and assume $\delta_{n} \in Z(L)$, where $Z(L)$ denotes the centre of $L$ and let $L_{0}:=K \delta_{n}$. We shall prove that $H^{1}\left(L_{0}, A\right)=0$. By Corollary 1.2 we can extend the action of $L$ to $A\left[Z_{1}, \ldots, Z_{n}\right]$ with the properties (i), (ii) of Corollary 1.3.

As before, we consider the exact sequence

$$
\begin{aligned}
0 \longrightarrow \int A^{L} \longrightarrow & A \longrightarrow A^{n^{2}}, \\
& a \rightsquigarrow\left(\delta_{i} \delta_{j}(a)\right)
\end{aligned}
$$

and put $B:=\exp \left(-Z_{1} \delta_{1}\right) \circ \cdots \circ \exp \left(-Z_{n} \delta_{n}\right)(A), \int A^{L}=\left\{a \in A \mid \delta(a) \in A^{L}\right.$ for all $\delta \in L\}$. Then the following commutative diagram has exact rows: 


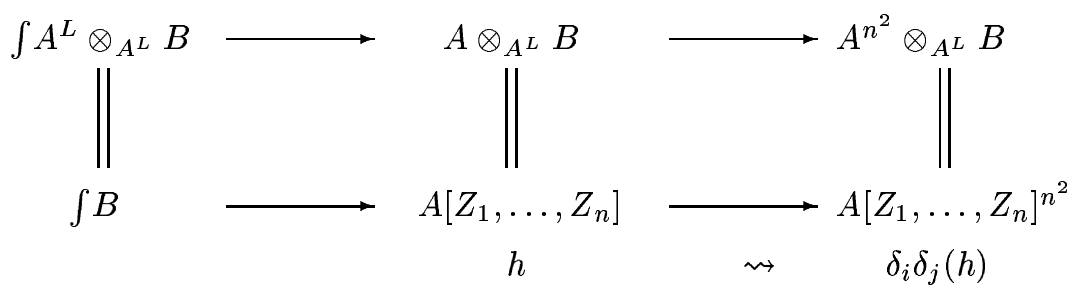

By assumption we have $Z_{n}=\sum \xi_{i} h_{i}, \xi_{i} \in A, h_{i} \in B$. As in the case $n=1$ (since $\delta_{i} \delta_{n}\left(Z_{n}\right)=\delta_{n} \delta_{i}\left(Z_{n}\right)=0$ ) we obtain a presentation of $Z_{n}$ with $\xi_{i} \in \int A^{L}$ and deduce $H^{1}\left(L_{0}, A\right)=0$ and $A^{L_{0}}[x]=A$ for a suitable $x \in A$.

Now $\bar{L}=L / L_{0}$ acts on $A^{L_{0}}$. In order to proceed by induction, we have to verify that

1. $\operatorname{Spec} A^{L_{0}} \longrightarrow \operatorname{Spec} A^{L}$ is faithfully flat,

2. $A^{L_{0}} \otimes_{A^{L}} A^{L_{0}} \longrightarrow A^{L_{0}}\left[Z_{1}, \ldots, Z_{n-1}\right]$ is an isomorphism.

The first property is clear because $A^{L_{0}} \subset A=A^{L_{0}}[x]$ is faithfully flat, and Spec $A \longrightarrow$ Spec $A^{L}$ is surjective.

Consider the following commutative diagram:

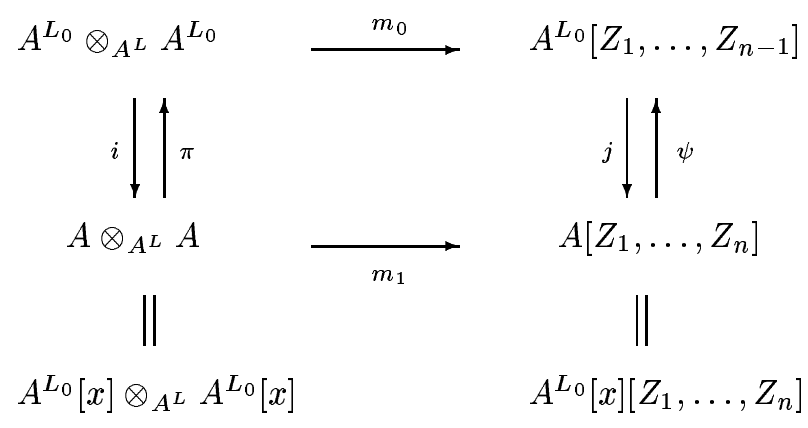

defined by

- $i(a \otimes b)=a \otimes b$,

- $j\left(h\left(Z_{1}, \ldots, Z_{n-1}\right)\right)=h\left(Z_{1}, \ldots, Z_{n-1}\right)$,

- $\pi(a(x) \otimes b(x))=a(0) \otimes b(0)$

- $\psi\left(h\left(x, Z_{1}, \ldots, Z_{n}\right)\right)=h\left(0, Z_{1}, \ldots, Z_{n-1},-\exp Z_{1} \delta_{1} \circ \cdots \circ \exp Z_{n-1} \delta_{n-1}(x)\right)$,

- $m_{0}(a \otimes b)=a \cdot \exp Z_{1} \delta_{1} \circ \cdots \circ \exp Z_{n-1} \delta_{n-1}(b)$,

- $m_{1}(a(x) \otimes b(x))=a(x) \cdot \exp Z_{1} \delta_{1} \circ \cdots \circ \exp Z_{n-1} \delta_{n-1} \circ \exp Z_{n} \delta_{n}(b(x))$. 
$m_{1}$ is an isomorphism by assumption, $\psi$ is obviously surjective and $i$ is injective. This implies that $m_{0}$ is an isomorphism and 2. is proved.

By induction hypothesis we obtain $H^{1}\left(L / L_{0}, A^{L_{0}}\right)=0$. Together with $A^{L_{0}}[x]=A$ this implies $H^{1}(L, A)=0$.

Corollary 2.2 Let $A$ be a commutative $K$-algebra, which we assume to be reduced. Let $L=\sum_{i=1}^{n} K \delta_{i} \subseteq \operatorname{Der}_{K}(A)$ be a nilpotent Lie-algebra and assume that the $\delta_{i}$ are locally nilpotent. Assume, moreover, that

1. A is a faithfully flat $A^{L}$-algebra.

2. The map $A \otimes_{K} A \longrightarrow A\left[Z_{1}, \ldots, Z_{n}\right]$ defined by $L$ is surjective.

Then $H^{1}(L, A)=0$.

Proof. We have to prove that the canonical map $A \otimes_{A^{L}} A \longrightarrow A\left[Z_{1}, \ldots, Z_{n}\right]$ is injective.

In [G-P1], proof of Proposition 1.6, we proved that there is a dense open subset $\cup D\left(f_{i}\right) \subseteq \operatorname{Spec} A, f_{i} \in A^{L}$ such that $\operatorname{Spec} A_{f_{i}} \longrightarrow \operatorname{Spec} A_{f_{i}}^{L}$ is a trivial quotient. This implies that

$$
\left(A \otimes_{A^{L}} A\right)_{f_{i}}=A_{f_{i}} \otimes_{A_{f_{i}}^{L}} A_{f_{i}} \longrightarrow A_{f_{i}}\left[Z_{1}, \ldots, Z_{n}\right]
$$

is an isomorphism.

Since $\cup D\left(f_{i}\right)$ is dense, we obtain that $A \otimes_{A^{L}} A \longrightarrow A\left[Z_{1}, \ldots, Z_{n}\right]$ is injective, which proves the corollary.

Corollary 2.3 Let $A$ and $L$ be as in Theorem 2.1 (respectively, moreover, that $A$ is reduced). Assume that the map $A \otimes_{A^{L}} A \longrightarrow A\left[Z_{1}, \ldots, Z_{n}\right]$ defined by $L$ is an isomorphism (respectively the map $A \otimes_{K} A \rightarrow A\left[Z_{1}, \ldots, Z_{n}\right]$ defined by $L$ is surjective) and $A$ is a flat $A^{L}$-algebra. Then there is an open subset $U \subseteq \operatorname{Spec} A^{L}$ such that Spec $A \longrightarrow U$ is a locally trivial geometric quotient.

Example. (cf. [D-F])

$$
A=K\left[x_{1}, x_{2}, y_{1}, y_{2}, z\right], \quad \delta=x_{1} \frac{\partial}{\partial x_{2}}+y_{1} \frac{\partial}{\partial y_{2}}+\left(1+x_{1} y_{2}^{2}\right) \frac{\partial}{\partial z} .
$$

We obtain $A^{\delta}=K\left[u_{1}, u_{2}, u_{3}, u_{4}, u_{5}\right]$ with $u_{1}=x_{1}, y=y_{1}, y_{3}=x_{1} y_{2}-$ $x_{2} y_{1}, u_{4}=3 y_{1} z-x_{1} y_{2}^{3}-3 y_{2}, u_{5}=3 x_{1}^{3} z-3 x_{1}^{2} x_{2} y_{2}^{2}+3 x_{1} x_{2}^{2} y_{1} y_{2}-x_{2}^{3} y_{1}-3 x_{1} x_{2}$, with relation $u_{2} u_{5}-u_{1}^{2} u_{4}-u_{3}^{3}-3 u_{1} u_{3}=0$.

We define $F(t):=(\exp t \delta)(z)=\frac{1}{3} x_{1} y_{1}^{2} t^{3}+x_{1} y_{1} y_{2} t^{2}+\left(1+x_{1} y_{2}^{2}\right) t+z$. Then $d:=9 \cdot \operatorname{disc}(F)=x_{1}\left(x_{1}^{2} y_{2}^{6}-6 x_{1} y_{1} y_{2}^{3} z+6 x_{1} y_{2}^{4}+9 y_{1}^{2} z^{2}-18 y_{1} y_{2} z+9 y_{2}^{2}\right)+4=$ $u_{1} u_{4}^{2}+4 \in A^{\delta}$ and we obtain that $B_{1}:=A_{d}[t] / F$ is étale over $A_{d} . \delta$ extends to $B_{1}$ by $\delta(t)=-1$ and then we have $B_{1}^{\delta}[t]=B_{1}$.

Altogether we see: Spec $A=D\left(x_{1}\right) \cup D(d)$, on the open set $D\left(x_{1}\right)$ a trivial quotient exists since $A_{x_{1}}^{\delta}\left[x_{2}\right]=A_{x_{1}}=: B_{2}$, but on $D(d)$ the quotient does 
not exist since over $D(d)$ we have fibres of different dimensions (here flatness fails, although the action is free in the sense of Mumford, cf. [D-F]). On the other hand, we have constructed an explicit étale covering $\left\{\operatorname{Spec} B_{1}\right.$, Spec $\left.B_{2}\right\}$ of Spec $A$ with $H^{1}\left(K \delta, B_{i}\right)=0$, as it should be, according to the conjecture of the introduction.

\section{References}

[C-E] Cartan, H; Eilenberg: Homological algebra. Princeton University Press, 1956.

[D-F] Deveney, J.K.; Finston, D.R.: A proper $G_{a}$ Action on $\mathbb{C}^{5}$ which is not locally trivial. Proc. AMS 123 (3), $651-655$ (1995).

[D-F-G] Deveney, J.K.; Finston, D.R.; Gehrke, M.: $G_{a}$ Actions on $\mathbb{C}^{n}$. Comm. Algebra, 22(12), 4977 - 4988 (1994).

[D-G] Demazure, M.; Gabriel, P.: Groupes Algébriques. North-Holland, Amsterdam 1970.

[G] Godement, R.: Introduction à la Théorie des Groupes de Lie. Publ. Math. de l'Université Paris VII, 1982.

[G-P1] Greuel, G.-M.; Pfister, G.: Geometric quotients of unipotent group actions. Proc. London Math. Soc. (3) 6775 - 105, 1993.

[G-P2] Greuel, G.-M.; Pfister, G.: Moduli spaces for torsion free modules on curve singularities. I. J. Algebraic Geometry 2, 81 - 135 (1993).

[G-H-P] Greuel, G.-M.; Hertling, C.; Pfister, G.: Moduli spaces of semiquasihomogeneous singularities with fixed principal part. Preprint 264, University of Kaiserslautern, 1995. To be published in J. Algebraic Geometry.

[H] Hadan, C.: Modulräume für ebene Kurvensingularitäten. Dissertation, Berlin 1996.

[K-M-T] Kambayashi, T.; Miyanishi, M.; Takeuchi, M.: Unipotent algebraic groups. Lecture Notes in Mathematics 414, Springer, Berlin 1974.

[L-P] Laudal, O.; Pfister, G.: Local moduli and singularities. Lecture Notes in Mathematics 1310 (Springer, Berlin, 1988).

[M-F] Mumford, D.; Fogarty, J.: Geometric invariant theory. 2nd enlarged edn, Ergebnisse der Mathematik und ihrer Grenzgebiete 34 (Springer, Berlin, 1982). 
[P] Popp, H.: Moduli Theory and Classification Theory. Lecture Notes in Mathematics 620 (Springer, Berlin 1977). 